\title{
THE FORMATION OF HIGH-MASS BLACK HOLES IN LOW-MASS X-RAY BINARIES
}

\author{
G. E. BROWN and C.-H. LEE \\ Department of Physics and Astronomy, State University of New York \\ Stony Brook, New York 11794-3800, USA \\ HANS A. BETHE \\ Floyd R. Newman Laboratory of Nuclear Studies, Cornell University \\ Ithaca, New York 14853, USA
}

\begin{abstract}
In this note we suggest that high-mass black holes; i.e., black holes of several solar masses, can be formed in binaries with low-mass main-sequence companions, provided that the hydrogen envelope of the massive star is removed in common envelope evolution which begins only after the massive star has finished He core burning. That is, the massive star is in the supergiant stage, which lasts only $\sim 10^{4}$ years, so effects of mass loss by He winds are small. Since the removal of the hydrogen envelope of the massive star occurs so late, it evolves essentially as a single star, rather than one in a binary. Thus, we can use evolutionary calculations of Woosley \& Weaver (1995) of single stars.

Using the Brown \& Bethe (1994) upper limit of $\sim 1.8 \mathrm{M}_{\odot}$ for the (gravitational) compact core mass that can evolve into a low-mass black hole, we find that high-mass black holes can be formed in the collapse of stars with ZAMS mass $\gtrsim 20 \mathrm{M}_{\odot}$. We somewhat arbitrarily take the upper limit for the evolution of the so-called transient sources to be $\sim 35 \mathrm{M}_{\odot}$ ZAMS mass. Mass loss by winds in stars sufficiently massive to undergo the LBV (luminous blue variable) stage may seriously affect the evolution of stars of ZAMS $>35-40 \mathrm{M}_{\odot}$, but we need calculations with improved mass loss rates before discussing these quantitatively. Both Portegies Zwart, Verbunt \& Ergma (1997) and Ergma \& van den Heuvel (1998) have suggested that roughly our chosen range of ZAMS masses must be responsible for the transient sources. We believe that the high-mass black hole limit of ZAMS mass $\sim 40 \mathrm{M}_{\odot}$ suggested by van den Heuvel \& Habets (1984) and later revised to $\geq 50 \mathrm{M}_{\odot}$ (Kaper et al. 1995) applies to massive stars in binaries, which undergo RLOF (Roche Lobe Overflow) early in their evolution. We will not pursue this here because calculations with improved
\end{abstract}


He-star mass loss rates by wind, now being carried out, are necessary before quantitative results can be obtained.

The most copious high-mass black holes of masses $\sim 6-7 \mathrm{M}_{\odot}$ have been found in the transient sources such as A0620. These have low-mass companions, predominantly of $\lesssim 1 \mathrm{M}_{\odot}$, such as $\mathrm{K}$ - or $\mathrm{M}$-stars. In the progenitor binaries the mass ratios must have been tiny, say $q \sim 1 / 25$. Normally such small ratios are thought to be rare; e.g. in binary evolution the companion distribution is often taken as $d q$, implying an very low probability of such a binary.

In this note we follow the evolutionary scenario for the black hole binary of de Kool et al. (1987). We show that the reason for this small $q$-value lies in the common envelope evolution of the binary. The smaller the companion mass, the greater the radius $R_{g}$ the giant must reach before its envelope meets the companion. This results because the orbit of a low-mass companion must shrink by a large factor in order to expel the envelope of the giant, hence the orbit must initially have a large radius. (Its final radius must be just inside its Roche Lobe, which sets a limit to the gravitational energy it can furnish.)

A large radius $R_{g}$ in turn means that the primary star must be in the supergiant stage. Thus it will have completed its He core burning while it is still "clothed" with hydrogen. This prevents excessive mass loss so that the primary retains essentially the full mass of its He core when it goes supernova. We believe this is why $\mathrm{K}$ - and $\mathrm{M}$-star companions of high-mass black holes are favored.

We find that the black holes in transient sources can be formed from stars with ZAMS masses in the interval $20-35 \mathrm{M}_{\odot}$. The black hole mass is only slightly smaller than the He core mass, typically $\sim 7 \mathrm{M}_{\odot}$.

Subject headings: black hole physics — X-rays: bursts — stars: binaries

\section{DEVELOPMENT}

Our evolutionary scenario is essentially the same as that of de Kool et al. (1987) for the black hole binary A0620-00. We apply this scenario somewhat schematically for the range of ZAMS $20-35 \mathrm{M}_{\odot}$ for the massive star. In Fig. 1 we show results of calculations of Fe core masses of single stars by Woosley \& Weaver (1995). Somewhere around ZAMS mass $20 \mathrm{M}_{\odot}$ the Fe core exceeds the Brown \& Bethe $(1994)$ limit of $\sim 1.8 \mathrm{M}_{\odot}$ for low-mass black-hole formation, so we take this as the beginning of high-mass black hole formation. 
The detailed behavior of this curve should not be taken seriously, but the large increase around $20-25 \mathrm{M}_{\odot}$ is of importance, as we discuss in detail later.

We wish to carry out a population synthesis so that we can estimate the number of transient sources. We make roughly the same assumptions as Bethe \& Brown $(1998,1999)$, our massive star $M_{B}$ lying in mass somewhere in between the O,B star progenitors of binary neutron stars and the progenitor of the massive black hole in Cyg X-1. Our low-mass companion is a main sequence star of mass $\sim 1 \mathrm{M}_{\odot}$. Thus, the ratio

$$
q=\frac{M_{A i}}{M_{B i}}
$$

is very small, and there will be great uncertainty in the initial number of binaries for such a small $q \sim 1 / 25$. We take the distribution as $d q$. The distribution in $q$ is unknown for such low-mass companions as are involved here, but our results will show that the flat distribution in $q$ is not unreasonable. We assume $\log a$, where $a$ is the semi-major axis of the orbit, to be uniformly distributed, over a total logarithmic interval of 7 . Thus, the fraction of binaries in a given interval of $\ln a$ is

$$
d \phi=\frac{d(\ln a)}{7}
$$

We take $\alpha$ the supernova rate to be $\sim 0.02$ per galaxy per year, somewhat larger than the 0.015 given by Cappellaro et al. (1997) and somewhat smaller than the 0.025 assumed by Bethe \& Brown (1998). The rate of supernovae (SN) is the same as the rate of birth of massive stars $M>10 \mathrm{M}_{\odot}$, so

$$
d \alpha=\alpha n\left(\frac{M}{10 \mathrm{M}_{\odot}}\right)^{-n} \frac{d M}{M}
$$

with $n$ the Salpeter exponent which we take to be 1.5. We furthermore assume a binarity of 0.5 . Once again, for our very small $q$ values this is uncertain.

We thus evolve as typical a ZAMS $25 \mathrm{M}_{\odot}\left(\right.$ star B) with companion $\sim 1 \mathrm{M}_{\odot}$ main sequence star (star A) as typical progenitor of the transient X-ray sources. The common envelope evolution can be done as in Bethe \& Brown (1998). With $M_{B_{i}}=25 \mathrm{M}_{\odot}$ and neglect of the accretion onto the main sequence mass $M_{A}$, we find from Bethe \& Brown

$$
\left(\frac{Y_{f}}{Y_{i}}\right)^{1.2}=\frac{1.2}{\alpha_{c e}} \frac{M_{B_{i}}}{M_{A}}
$$

where $Y=M_{B} / a$. Here the coefficient of dynamical friction $c_{d}$ was taken to be 6 , in the range of the 6-8 for supersonic flow with Mach number 3-10 (Ruffert 1994; Ruffert \& 
Arnett 1994). The result is relatively insensitive to $c_{d}$, the exponent 1.2 resulting from $1+1 /\left(c_{d}-1\right)$.

Thus, in our case

$$
\frac{Y_{f}}{Y_{i}}=17\left(\frac{\alpha_{c e} M_{A}}{M_{\odot}}\right)^{-0.83}=30\left(\frac{0.5}{\alpha_{c e}} \frac{M_{\odot}}{M_{A}}\right)^{0.83}
$$

We expect $\alpha_{c e} \simeq 0.5$, under the assumption that the kinetic energy of the expelled envelope is equal to that it originally possessed in the massive star, but it could be smaller. From this we obtain

$$
\frac{a_{i}}{a_{f}}=\frac{M_{B_{i}} Y_{f}}{M_{B_{f}} Y_{i}}=90\left(\frac{0.5}{\alpha_{c e}} \frac{M_{\odot}}{M_{A}}\right)^{0.83},
$$

where we have taken the He star mass $M_{B_{f}}$ to be $1 / 3$ of $M_{B_{i}}$. In order to survive spiral-in, the final separation $a_{f}$ must be sufficient so that the main sequence star lies at or inside its Roche Lobe, about $0.2 a_{f}$ if $M_{A}=\mathrm{M}_{\odot}$. This sets $a_{f} \sim 5 R_{\odot}=3.5 \times 10^{11} \mathrm{~cm}$ and

$$
a_{i}=3.15\left(\frac{0.5}{\alpha_{c e}}\right)^{0.83} \times 10^{13} \mathrm{~cm} .
$$

$\sim 2$ A.U.. This exceeds the radius of the red giant tip in the more numerous lower mass stars in our interval, so the massive star must generally be in the supergiant phase where it meets the main sequence star, i.e., the massive star must be beyond He core burning. E.g., the red giant tip (before the He core burning) for a $20 \mathrm{M}_{\odot}$ star is at $0.96 \times 10^{13} \mathrm{~cm}$, for a $25 \mathrm{M}_{\odot}$ star, $2.5 \times 10^{13} \mathrm{~cm}$ (Schaller et al. 1992). These numbers are, however, somewhat uncertain. Notice that decreasing $\alpha_{c e}$ will increase $a_{i}$. Decreasing $M_{A}$ has little influence, because with the smaller stellar radius the minimum $a_{f}$ will decrease nearly proportionately. Note that neglect of accretion onto the main sequence star would change the exponent 0.83 to unity, so accretion is unimportant except in increasing the final mass.

Now a ZAMS $25 \mathrm{M}_{\odot}$ star ends up at radius $6.7 \times 10^{13} \mathrm{~cm}\left(\sim 2 a_{i}\right)$ following $\mathrm{He}$ shell burning (Weaver, Zimmerman \&Woosley 1978). Thus the interval between $a_{i}$ and $6.7 \times 10^{13} \mathrm{~cm}$ is available for spiral-in without merger' so that a fraction

$$
\frac{1}{7} \ln \left(\frac{6.7}{3.15\left(\frac{0.5}{\alpha_{c e}}\right)^{0.83}}\right) \simeq 0.11
$$

\footnotetext{
${ }^{1}$ Note that envelope removal does not occur at the Roche Lobe on the thermal time scale $\tau_{t h}$ but at the low-mass star since the remaining lifetime of the giant is $\sim 10^{4} \mathrm{yrs}$, much shorter than $\tau_{t h}$.
} 
of the binaries survive spiral-in, but are close enough so that the main sequence star is encountered by the evolving $\mathrm{H}$ envelope of the massive star. The He core burning will be completed before the supergiant has moved out to $\sim 2$ A.U., so binaries which survive spiral-in will have He cores which burn as "clothed", namely as in single stars.

\section{BIRTH RATE OF TRANSIENT SOURCES}

Given our assumptions in Section 1, the fraction of supernovas which arise from ZAMS stars between 20 and $35 \mathrm{M}_{\odot}$ is

$$
\frac{1}{2^{3 / 2}}-\frac{1}{3.5^{3 / 2}}=0.20
$$

where we have assumed the mass $10 \mathrm{M}_{\odot}$ is necessary for a star to go supernova. A Salpeter function with index $n=1.5$ is assumed here. Our assumption that the binary distribution is as $d q$ is arbitrary, and gives us a factor $1 / 25$ for a $1 \mathrm{M}_{\odot}$ companion. Note, however, that had we included higher mass companions, the change in the final projected number of transient sources would not be order of magnitude, because the hydrogen burning time goes inversely as mass squared. Thus, for supernova rate 2 per century, our birth rate for transient sources in the Galaxy is

$$
2 \times 10^{-2} \times 0.5 \times 0.11 \times 0.20 \times 0.04 \simeq 8.8 \times 10^{-6} \mathrm{yr}^{-1}
$$

where 0.5 is the assumed binarity, 0.11 comes from eq. (1.8), and the final (most uncertain) factor 0.04 results from a distribution flat in $q$ and an assumed $1 \mathrm{M}_{\odot}$ companion star.

In order to estimate the number of transient sources with black holes in the Galaxy, we should know the time that a main sequence star of mass $\sim 1 \mathrm{M}_{\odot}$ transfers mass to a more

massive companion. For a main-sequence donor, the mass transfer rate is $\sim 10^{-10} \mathrm{M}_{\odot} \mathrm{yr}^{-1}$, almost independent of donor mass (Verbunt \& van den Heuvel 1995). As mass is transferred, the mass of the donor decreases and with it the radius of the donor. Quite a few low-mass $\mathrm{X}$-ray binaries have X-ray luminosities that imply accretion rates in excess of $10^{-10} \mathrm{M}_{\odot} \mathrm{yr}^{-1}$, leading to suggestions of additional mechanisms for loss of angular momentum from the binary, to increase mass transfer. Verbunt \& Zwaan (1981) estimate that magnetic braking can boost the transfer of mass in a low-mass binary. We somewhat arbitrarily adopt an effective mass transfer rate of $10^{-9} \mathrm{yr}^{-1}$ for main sequence stars and $10^{-8} \mathrm{yr}^{-1}$ for the two systems that have subgiant donors (V404 Cyg and XN Sco 94). In order to estimate the number of high-mass black hole, main sequence star binaries in the Galaxy we should multiply the birth rate (2.2) times the $10^{9}$ yrs required, at the assumed mass loss rate, to strip the main sequence star, obtaining 8800 as our estimate. Not all of these will fill their 
Roche Lobes. Those that do not may not now be visible, but will be later, as they begin evolving as one of those with subgiant donors. The fact that two of seven observed binaries are subgiants, although the lifetime of the latter is two orders of magnitude less than the main sequence lifetime, suggests that some fraction of our 8800 estimated binaries do not fill their Roche Lobes. From the observed black-hole transient sources Wijers (1996) arrives at 3000 low-mass black hole sources in the Galaxy, but regards this number as a lower limit. Beginning from this, which we regard as an observational estimate, we note that the two subgiants in Table 1 involve the more massive F,G and A stars. These indicate an $\sim 100$ times greater population of unevolved main sequence stars in this range which lie quietly inside their Roche Lobes. Thus, including the quiescent binaries might give as many as $(2 / 7) \times 100 \times 3000$ or $\sim 10^{5}$ additional binaries, suggesting that our above estimate may be an order of magnitude too low. Estimates of the number of transient sources are very uncertain, but it is clear that there are orders of magnitude more of them than of the Cygnus X-1 type objects with high-mass black hole and massive star companion.

If we assume that ZAMS masses $\sim 10-18 \mathrm{M}_{\odot}$ evolve into a neutron star, we should have $\sim 3$ times more neutron stars than high-mass black holes (see eq. (2.1)). The upper limit follows from our belief that SN $(1987 \mathrm{~A})$ with progenitor $\sim 18 \mathrm{M}_{\odot}$ ZAMS went into a low-mass black hole, following the scenario of Brown \& Bethe (1994). On the basis of a Monte Carlo calculation using the kick velocities of Cordes \& Chernoff (1997) we find that $\sim 1 / 2$ of the binaries containing He-star, low-mass main sequence companion (with $M \simeq 1 \mathrm{M}_{\odot}$ ) will be disrupted in the explosion. Thus, we find only a slightly higher birth rate for LMXB's with neutron stars than with black holes, although the numbers could be equal to within our accuracy. The LMXB's with neutron stars tend to be much brighter than those with black holes, indicating an order of magnitude greater transfer rate. With the correspondingly shorter main-sequence lifetime, this would give us several hundred LMXBs with neutron stars, a factor of several greater than the observed number, $\sim 130$. Given the lifetime of $\sim 10^{8}$ years of a LMXB and accretion at roughly the Eddington rate of $\sim 10^{-9}-10^{-8} \mathrm{M}_{\odot} \mathrm{yr}^{-1}$, it is reasonable that some neutron stars accrete a reasonable fraction of a solar mass $\mathrm{M}_{\odot}$ (van den Heuvel 1995). We expect the masses of these to exceed the Brown \& Bethe (1994) limit of $1.5 \mathrm{M}_{\odot}$ for maximum neutron star mass, and evolve into low-mass black holes. The low-mass black-hole, low-mass main sequence star systems would not be seen. 


\section{ESTIMATED MASSES OF THE BLACK HOLES IN TRANSIENT SOURCES}

As we showed below eq. (1.7), the He core of the massive star will in general be uncovered only after He core burning is completed. The remaining time for He burning (in a shell) will be short, e.g., for a $20 \mathrm{M}_{\odot}$ ZAMS star it is only $1.4 \times 10^{4}$ years (Schaller et al. 1992). Therefore the mass loss by wind after uncovering the He core will not be large, and when the star finally becomes a supernova, its mass will be almost equal to the He core of the original star. The latter can be calculated from

$$
M_{H e} \simeq 0.08\left(M_{Z A M S}\right)^{1.4}
$$

so for ZAMS masses $20-35 \mathrm{M}_{\odot} M_{H e}$ will lie in the interval $\sim 5.3-11.6 \mathrm{M}_{\odot}$. In fact, the lower limit looks a bit small, because the He core of the $\sim 18 \mathrm{M}_{\odot}$ progenitor of 1987A is generally taken as $\sim 6 \mathrm{M}_{\odot}$.

Bailyn et al. (1998) find the black hole masses in transient sources to be clustered about $\sim 7 \mathrm{M}_{\odot}$, except for V404 Cyg which has a higher mass. This is in general agreement with our scenario, because most of the black holes will come from the more numerous stars of ZAMS mass not far from our lower limit of $\sim 20 \mathrm{M}_{\odot}$. Two points are important to note:

1. Not much mass can have been lost by wind. Naked He stars have rapid wind loss. However in our scenario the He star is made naked only during He shell burning and therefore does not have much time $\left(\lesssim 10^{4}\right.$ yrs $)$ to lose mass by wind.

2. There are good reasons to believe that the initial He core will be rotating (cf. Mineshige, Nomoto \& Shigeyama 1993). The way in which the initial angular momentum affects the accretion process has been studied by Mineshige et al. (1997) for black hole accretion in supernovae. In general accretion discs which are optically thick and advection dominated are formed. The disc is hot and the produced energy and photons are advected inward rather than being radiated away. The disc material accretes into the black hole at a rate of $>10^{6} \dot{M}_{E d d}$ for the first several tens of days. Angular momentum is advected outwards. Our results show that little mass is lost, because the final $\sim 7 \mathrm{M}_{\odot}$ black hole masses are not much less massive than the He core masses of the progenitors, and some mass is lost by wind before the core collapses. The latter loss will not, however, be great, because there is not much time from the removal of the He envelope until the collapse.

Accretion of the He into the black hole will differ quantitatively from the above, but we believe it will be qualitatively similar. The fact that the helium must be advected inwards 
and that little mass is lost as the angular momentum is advected outwards is extremely important to establish. This is because angular momentum, essentially centrifugal force, has been suggested by Chevalier (1996) to hold up hypercritical accretion onto neutron stars in common envelope evolution. (Chevalier (1993) had first proposed the hypercritical accretion during this evolutionary phase to turn the neutron stars into black holes, the work followed up by Brown (1995) and Bethe \& Brown (1998).) However, once matter is advected onto a neutron star, temperatures $\gtrsim 1 \mathrm{MeV}$ are reached so that neutrinos can carry off the energy. The accreted matter simply adds to the neutron star mass, evolving into an equilibrium configuration. Thus, this accretion does not differ essentially from that into a black hole. In either case of neutron star or black hole an accretion disc or accretion shock, depending on amount of angular momentum, but both of radius $\sim 10^{11} \mathrm{~cm}$, is first formed, giving essentially the same boundary condition for the hypercritical accretion in either case, black hole or neutron star. Thus, the masses of the black holes in transient sources argue against substantial inhibition of hypercritical accretion by jets, one of the Chevalier (1996) suggestions.

Measured mass functions, which give a lower limit on the black hole mass are given in Table 1. Only GRO J0422+32 and 4U 1543-47 have a measured mass function $\lesssim 3 \mathrm{M}_{\odot}$. Results of Callanan et al. (1996) indicate that the angle $i$ between the orbital plane and the plane of the sky for GRO J0422+32 is $i<45^{\circ}$, and recent analysis by Orosz et al. (1998) indicate that the angle $i$ for $4 \mathrm{U} 1543-47$ is $20^{\circ}<i<40^{\circ}$. So both GRO J0422+32 and $4 \mathrm{U}$ 1543-47 also contain high-mass black holes.

\section{GENERAL DISCUSSION}

There is agreement (Portegies Zwart et al. 1997; Ergma \& van den Heuvel, 1998) that in order to make enough transient sources the progenitors of the black holes must begin at relatively low masses, $\sim$ ZAMS $20 \mathrm{M}_{\odot}$. We take the upper limit somewhat arbitrarily to be $\sim 35 \mathrm{M}_{\odot}$ about where rapid mass loss occurs and stars may enter into the LBV phase. Our upper limit is relatively unimportant since most of the stars considered will lie near the lower limit.

Based on the observations of Kaper et al. (1995) that the companion is a hypergiant, Ergma \& van den Heuvel (1998) argue that the progenitor of the neutron star in 4U1223-62 must have a ZAMS mass $\gtrsim 50 \mathrm{M}_{\odot}$. Brown, Weingartner \& Wijers (1996), by similar argumentation, arrived at $\sim 45 \mathrm{M}_{\odot}$, but then had the difficulty that 4U1700-37, which they suggested to contain a low-mass black hole appeared to evolve from a lower mass star than the neutron star in 1223. Wellstein \& Langer (1999) suggest the alternative that in 
1223 the mass occurs in the main sequence phase (Case A mass transfer) which would be expected to be quasi conservative. They find that the progenitor of the neutron star in 1223 could come from a mass as low as $26 \mathrm{M}_{\odot}$. This is in agreement with Brown et al. (1996) for conservative mass transfer (their Table 1), but these authors discarded this possibility, considering only RLOF (Case B mass transfer) in which case considerable mass would be lost.

Wellstein \& Langer (1999) are in agreement with Brown et al. (1996) that 4U1700-37 should come from a quite massive progenitor. Conservative evolution here is not possible because of the short period of 3.4 days (Rubin et al. 1996). The compact object mass is here $1.8 \pm 0.4 \mathrm{M}_{\odot}$ (Heap \& Corcoran 1992). Brown et al. (1996) suggest that the compact object is a low-mass black hole. The upper mass limit for these was found by Brown \& Bethe (1994) to be $\sim 1.8 \mathrm{M}_{\odot}$, as compared with an upper limit for neutron star masses of $\sim 1.5 \mathrm{M}_{\odot}$. Thus, there seems to be evidence for some ZAMS masses of $\sim 40-50 \mathrm{M}_{\odot}$ ending up as low-mass compact objects, whereas we found that lower mass stars in the interval from $\sim 20-35 \mathrm{M}_{\odot}$ ended up as high-mass black holes. In this sense we agree with Ergma \& van den Heuvel (1998) that low-mass compact object formation "is connected with other stellar parameters than the initial stellar mass alone." We suggest, however, following Brown et al. (1996) that stars in binaries evolve differently from single stars because of the different evolution of the He core in binaries resulting from RLOF in their evolution. Namely, "naked" He cores evolve to smaller final compact objects than "clothed" ones.

In fact, this different evolution of binaries was found by Timmes, Woosley \& Weaver (1996). They pointed out that stars denuded of their hydrogen envelope in early RLOF in binaries would explode as Type Ib supernovae. They found the resulting remnant gravitational mass following explosion to be in the interval of $1.2-1.4 \mathrm{M}_{\odot}$, whereas in exploding stars of all masses with hydrogen envelope (Type II supernova explosion) they found a peak at about $1.28 \mathrm{M}_{\odot}$, chiefly from stars of low masses and another peak at $1.73 \mathrm{M}_{\odot}$ more from massive stars. Our Fe core masses in Fig. 四 come from essentially the same calculations, but the "Remnant" masses of Woosley \& Weaver (1995) are somewhat greater than those used by Timmes et al. (1996). In fact, the differences between the masses we plot and those of Timmes et al. come in the region $\sim 1.7-1.8 \mathrm{M}_{\odot}$ (gravitational). This is just in the Brown \& Bethe (1994) range for low-mass black holes. It may be that some of the stars with low-mass companions evolve into low-mass black holes. Presumably these would give lower luminosities than the high-mass black holes, although at upper end of the mass range we discuss 4U1700-37 seems to be an example of such a system. Of course here the high luminosity results from the high mass loss rate of the giant companion. There are substantial ambiguities in fallback, etc., from the explosion. Our point in this paper is that most of the higher mass single stars $20-35 \mathrm{M}_{\odot}$ go into high mass black holes. (The Brown 
\& Bethe (1994) limit for low-mass black hole formation is $\sim 1.5-1.8 \mathrm{M}_{\odot}$ gravitational, but there is some give and take in both lower and upper limit. Also the stars are not all the same. In particular different metallicities will give different wind losses.)

In determining the upper mass limit for which a low-mass compact object can result from binary evolution with RLOF (Case B mass transfer) the Brown et al. (1996) scenario must be revised because it is now realized that He-star wind loss rates employed by Woosley, Langer \& Weaver (1995) were too high. With lower rates the differences in behavior between "clothed"" and "naked" He core evolutions will be diminished because much of the difference arises from the mass losses and how it affects the convective ${ }^{12} C$ burning as we discuss below.

In the mass range $\sim 20-35 \mathrm{M}_{\odot}$, the compact objects resulting from naked He stars (Type Ib SN explosions) are sufficiently far below the maximum neutron star mass that they will still remain neutron stars when better (lower) He star mass loss rates are employed. Indeed, for our $25 \mathrm{M}_{\odot}$ star, Wellstein and Langer (1999) find that halving the mass loss rate lowers the mass of the CO core by only $2.5 \%$. However, at about the upper end of this range of masses, the outcome may, indeed, be changed. We will somewhat arbitrarily focus on the mass region $\sim 40 \mathrm{M}_{\odot}$, studying how lower mass loss rates might affect the outcome.

Until recently W.-R. wind loss rates were taken from observed winds which originated chiefly from free-free scattering. These depend quadratically on density. Because of "clumpiness" in the winds, the mass loss rate was overestimated. Polarization measurements of the Thomson scattering, which depend linearly on the wind, give substantially lower mass rates, in approximate agreement with the rates that would be deduced from the observed rate of increase in orbital periods for spherical mass loss

$$
\frac{\dot{M}}{M}=\frac{2 \dot{P}}{P}
$$

In V444 Cygni $\dot{P}=0.202 \pm 0.018 s \mathrm{yr}^{-1}$ was obtained by Khaliullin et al. (1984) and $M_{W R}=9.3 \pm 0.5 \mathrm{M}_{\odot}$ by Marchenko et al. (1994), resulting in

$$
\dot{M}_{d y n}=1.03 \times 10^{-5} \mathrm{M}_{\odot} \mathrm{yr}^{-1} .
$$

This is to be compared with the

$$
\dot{M}=0.75 \times 10^{-5} \mathrm{M}_{\odot} \mathrm{yr}^{-1}
$$

obtained by St.-Louis et al. (1993). In later work Moffat \& Robert (1994) arrive at a mean of $(0.7 \pm 0.1) \times 10^{-5} \mathrm{M}_{\odot} \mathrm{yr}^{-1}$. The mass loss rate employed by Woosley, Langer \& Weaver (1995) was that of Langer (1989). Specifically, $\dot{M}=-k M^{2.5}$, with $M$ in $\mathrm{M}_{\odot}$ and $\dot{M}$ in 
$\mathrm{M}_{\odot} \mathrm{yr}^{-1}$ and $k=6 \times 10^{-8}$ as long as the carbon surface mass fraction does not exceed 0.02 and $k=10^{-7}$ afterwards. Choosing an average of $k=8 \times 10^{-8}$ we find the WLW rate to be $2.1 \times 10^{-5} \mathrm{M}_{\odot} \mathrm{yr}^{-1}$, a factor of 2 larger than $\dot{M}_{d y n}$. Given the many uncertainties in our estimate, we feel the range of two to three times less than the Langer (1989) mass loss rate to be reasonable. It should also be remembered that stars vary substantially in metallicity, and that there will be a range of variation even of those in the Galactic disc. Of course stars in the metal poor Magellanic clouds should have substantially lower winds.

In the region of ZAMS $\sim 40 \mathrm{M}_{\odot}$ calculation of Wellstein \& Langer (1999) show that both the final He core mass and $\mathrm{C} / \mathrm{O}$ core mass increase $\sim 23 \%$ in the mass loss rate is halved. Thus, reducing the mass loss rate by a factor of 2 does not increase the final mass by a similar factor. The main reason is that if the mass loss is reduced, then the He stars remain somewhat more massive, thus also more luminous and therefore have a higher mass loss rate than had their mass been reduced earlier. There are also other, less important, feedbacks.

In the case of $\sim 60 \mathrm{M}_{\odot}$ stars, the He core and $\mathrm{C} / \mathrm{O}$ cores increase $\sim 31 \%$ if the mass loss rate is halved and a factor of $\sim 1.8-1.9$ if it is cut by $1 / 4$. In fact, in this case, a reasonable interpolation formula for these lower mass loss rates is

$$
\frac{M}{M_{0}}=(1.33)^{1 / 2 f}
$$

where $M_{0}$ was calculated with the Langer (1989) rates and $f$ is the fractional decrease in winds from these rates. Wellstein \& Langer (1999) also evolve a $36 \mathrm{M}_{\odot}$ He core, which would come from an $\sim 85 \mathrm{M}_{\odot}$ ZAMS star, with $f=1 / 4$ of the Langer et al (1989) wind loss rates. Their final 7.5 $\mathrm{M}_{\odot}$ He star mass is not enough for Cyg X-1. However, Woosley, Langer \& Weaver (1993) with the Langer 1989 mass loss rate found a $9.71 \mathrm{M}_{\odot}$ He core in evolving a ZAMS $85 \mathrm{M}_{\odot}$ star with mass loss. In the latter case the WNL phase lasted $>1 / 3$ of the W.-R. phase; i.e., the He core initially had some hydrogen envelope. This illustrates the order of uncertainties that may come in the evolution of very massive stars, arising from mass loss in the LBV phase.

Although the final He core and $\mathrm{C} / \mathrm{O}$ core scale by roughly the same factor the $\mathrm{Fe}$ core, crucial for the compact object masses, is not expected to do the same. Firstly, rather trivially, even if the baryon number Fe core did scale in the same way, one would expect corrections downwards in core mass from binding energy correction. These decreases in gravitational mass would be greater for higher mass cores, so the Fe cores would scale somewhat less rapidly than the $\mathrm{C} / \mathrm{O}$ cores.

More important for the mass of the Fe core is the ZAMS mass at which the convective carbon burning is skipped, because, as seen in the single stars in Fig. 1, a big jump in Fe 
core masses occurs here.

The convective carbon burning phase (when it occurs) is extremely important in presupernova evolution, because this is the first phase in which a large amount of entropy can be carried off in $\nu \bar{\nu}$-pair emission, especially because this phase is of long duration. The reaction in which carbon burns is ${ }^{12} C(\alpha, \gamma){ }^{16} O$ (other reactions like ${ }^{12} C+{ }^{12} C$ would require excessive temperatures). The cross section of ${ }^{12} C(\alpha, \gamma){ }^{16} O$ is still not accurately determined; the lower this cross section the higher the temperature of the ${ }^{12} \mathrm{C}$ burning, and therefore the more intense the $\nu \bar{\nu}$ emission. With the relatively low ${ }^{12} C(\alpha, \gamma){ }^{16} O$ rates ${ }^{2}$ determined both directly from nuclear reactions and from nucleosynthesis by Weaver \& Woosley (1993), the entropy carried off during ${ }^{12} \mathrm{C}$ burning in the stars of ZAMS mass $\leq 18 \mathrm{M}_{\odot}$ is substantial. The result is rather low-mass Fe cores for these stars, which can evolve into neutron stars. Note that in the literature earlier than Weaver \& Woosley (1993) often large ${ }^{12} C(\alpha, \gamma){ }^{16} O$ rates were used, so that the ${ }^{12} C$ was converted into oxygen and the convective burning did not have time to be effective. Thus its role was not widely appreciated.

Of particular importance is the ZAMS mass at which the convective carbon burning is skipped. In the Woosley \& Weaver (1995) calculations this occurs for single stars at ZAMS mass $19 \mathrm{M}_{\odot}$ but with a slightly lower ${ }^{12} \mathrm{C}(\alpha, \gamma){ }^{16} \mathrm{O}$ rate it might come at $20 \mathrm{M}_{\odot}$ or higher (Brown 1997). As the progenitor mass increases, it follows from general polytropic arguments that the entropy at a given burning stage increases. At the higher entropies of the more massive stars the density at which burning occurs is lower, because the temperature is almost fixed for a given fuel. Lower densities decrease the rate of the triple- $\alpha$ process which produces ${ }^{12} C$ relative to the two-body ${ }^{12} C(\alpha, \gamma){ }^{16} O$ which produces oxygen. Therefore, at the higher entropies in the more massive stars the ratio of ${ }^{12} \mathrm{C}$ to ${ }^{16} \mathrm{O}$ at the end of He burning is lower. The star skips the long convective carbon burning and goes on to the much shorter oxygen burning. Oxygen burning goes via ${ }^{16} \mathrm{O}+{ }^{16} \mathrm{O}$ giving various products, at very much higher temperature than ${ }^{12} C(\alpha, \gamma){ }^{16} O$ and much faster. Since neutrino cooling during the long carbon-burning phase gets rid of a lot of entropy of the core, skipping the convective carbon burning phase leaves the core entropy higher and the final Chandrasekhar core fatter.

In Fig. 1 the large jump in compact object mass in single stars at ZAMS mass $\sim 19 \mathrm{M}_{\odot}$ is clearly seen. From our discussion in the last section we see that this is just about at the

\footnotetext{
${ }^{2}$ Weaver \& Woosley use $S(E)=S(300)=170 \mathrm{keV}$ barns, remarkably close to the $169 \pm 55 \mathrm{keV}$ barns arrived at by Barnes (1995). Given the large uncertainty (stemming chiefly from that in the E2 rate, the good agreement may be somewhat accidental.
} 
point where our Fe core mass goes above $\sim 1.8 \mathrm{M}_{\odot}$ and, therefore, above this mass one would expect single stars to go into high-mass black holes. Arguments have been given that SN (1987A) with progenitor ZAMS mass of $\sim 18 \mathrm{M}_{\odot}$ evolved into a low-mass black hole (Brown \& Bethe 1994). We believe from our above arguments and Fig. 1 that soon above the ZAMS mass of $\sim 18 \mathrm{M}_{\odot}$, single stars go into high-mass black holes without return of matter to the Galaxy. Thus, the region of masses for low-mass black hole formation in single stars is narrow. The precise upper mass limit is not clear, but certainly in the range of $\gtrsim 20 \mathrm{M}_{\odot}$ ZAMS. This is in agreement with Wellstein \& Langer (1999) who find a minimum black hole progenitor mass for single stars of $21 \mathrm{M}_{\odot}$.

Thus far our discussion has been chiefly about single stars, in which the He burns while clothed by a hydrogen envelope.

In binary evolution, if the hydrogen envelope is removed before the helium core burning in either Case A or Case B (RLOF) mass transfer, the resulting "naked" He star burns quite differently from a "clothed" one. Weaver \& Woosley (1993) find that the convective carbon burning tends to be skipped when the central ${ }^{12} C$ abundance at the end of helium core burning is less than $\sim 15 \%$. In Woosley, Langer \& Weaver (1995) with the Langer (1989) helium mass loss rate, this central ${ }^{12} \mathrm{C}$ abundance is $34 \%$ for a ZAMS $40 \mathrm{M}_{\odot}$ star. With mass loss rate decreased to half, it is still 33\% (Wellstein \& Langer, 1999) hardly changed. However, as noted earlier, the CO mass for a ZAMS $40 \mathrm{M}_{\odot}$ star is increased from $2.33 \mathrm{M}_{\odot}$ to $2.87 \mathrm{M}_{\odot}$ with halved mass loss rate. Provisionally Wellstein \& Langer (1999) have suggested that the magnitude of the CO mass chiefly determines the fate of the star, and that the $2.87 \mathrm{M}_{\odot} \mathrm{CO}$ core could go into a black hole. However, halving the mass loss rate of the naked He star decreases the central carbon abundance at the end of He core burning hardly at all. Thus, there will still be a long period of ${ }^{12} C$ convective core burning. As seen from Fig. 1 for less massive stars in the mass region $\sim 20 \mathrm{M}_{\odot}$, this can easily lower the compact core mass by $\sim 0.5 \mathrm{M}_{\odot}$. On the other hand, for each $1 \mathrm{M}_{\odot}$ added to the He envelope at the envelope at the time of SN explosion, an additional $\sim 3 \times 10^{49} \operatorname{ergs}$ is needed to expel it in either a prompt or delayed supernova explosion. This is $\sim 2 \%$ of the SN explosion energy, so would work towards formation of a high-mass black hole.

Given the increase in He and $\mathrm{CO}$ cores from the decreased mass loss rates, we estimate that stars of ZAMS masses $\sim 40 \mathrm{M}_{\odot}$ which lose their $\mathrm{H}$ envelopes by wind will end up as low-mass black holes, 1700-37 being an example. The possible mass ranges in which this can happen cannot be determined until the dynamic evolution of the CO cores formed with the lowered He-star wind rates of Wellstein \& Langer (1999) is carried out.

It seems clear that with the lower metallicity in the LMC and consequently lower mass loss rates that the ${ }^{12} \mathrm{C}$ convective core burning will not be skipped so the Fe cores in the 
$\gtrsim 40 \mathrm{M}_{\odot}$ region of ZAMS masses will be larger, and will evolve into black holes. This may help to explain why there are two HMXB's with high-mass black holes, LMC X-1 and LMC $\mathrm{X}-3$, in the LMC, whereas Cyg X-1 is the only clear example in the disc.

\section{CONCLUSION}

We have shown that it is likely that single stars in the range of ZAMS masses $\sim 20-35 \mathrm{M}_{\odot}$ evolve into high-mass black holes without return of matter to the Galaxy. This results because at mass $\sim 20 \mathrm{M}_{\odot}$ the convective carbon burning is skipped and this leads to substantially more massive Fe cores. Even with more realistic reduced mass loss rates on He stars, it is unlikely that stars in this mass range in binaries evolve into high-mass black holes, because the progenitor of the compact object when stripped of its hydrogen envelope in either Case A (during main sequence) or Case B (RLOF) mass transfer will burn as a "naked" He star, ending up as an Fe core which is not sufficiently massive to form a high-mass black hole.

In the region of ZAMS mass $\sim 40 \mathrm{M}_{\odot}$, depending sensitively on the rate of He-star wind loss, the fate of the primary in a binary may be a low-mass black hole. We are unable to pin down the limit for high-mass black hole formation until better mass loss rates are determined.

In our estimates we have assumed the Brown \& Bethe (1994) estimates of $1.5 \mathrm{M}_{\odot}$ for maximum neutron star mass and $1.5-1.8 \mathrm{M}_{\odot}$ for the range in which low-mass black holes can result.

In our evolution of the transient sources using Case $\mathrm{C}$ (during He shell burning) mass transfer, almost the entire He core will collapse into a high-mass black hole, explaining the more or less common black hole mass of $\sim 7 \mathrm{M}_{\odot}$ for these objects, with the possible exception of V404 Cygni where the mass may be greater. Our evolution gives an explanation for the seemingly large gap in black-hole masses, between the $\gtrsim 1.5 \mathrm{M}_{\odot}$ for the black hole

we believe was formed in 1987A and the $\sim 1.8 \mathrm{M}_{\odot}$ black hole we suggest in 1700-37 and the $\sim 7 \mathrm{M}_{\odot}$ in the transient sources.

We note that following the removal of the $\mathrm{H}$ envelope by Case $\mathrm{C}$ mass transfer, the collapse inwards of the He envelope into the developing black hole offers the Collapsar scenario for the most energetic gamma ray bursters of Woosley (1993) and MacFadyen \& Woosley (1999). Especially if our higher estimate of $\sim 10^{5}$ of the high-mass black-hole, main-sequence star binaries is roughly correct, a simple estimate (Brown, Lee \& Wijers 1999) shows that they would be the largest population of possible progenitors. 
We dedicate this work to Dave Schramm, a giant among astrophysicists. One of the authors (G.E.B.) invited him to edit the astrophysics section of Physics Reports and enjoyed his collegiality for many years.

We are especially grateful to S. Wellstein and N. Langer for communicating their results with modified mass loss rates to us before publication. We would like to thank Charles Bailyn for useful discussions and, especially, for putting the data in Table 1 together for us. We would like to thank Stan Woosley, who not only provided us with most of the results we used, but also clarified their interpretation in several communications. We also thank Adam Burrows for a clear explanation of the consequences of skipping the convective carbon burning. We wish to thank Simon Portegies Zwart for several helpful discussions. We were supported by the U.S. Department of Energy under Grant No. DE-FG02-88ER40388.

\section{REFERENCES}

Bailyn, C. D., Jain, R. K., Coppi, P., \& Orosz, J. A. 1998, ApJ, 499, 367

Barnes, C.A. 1995, Nucl. Phys. A, 588, 295c

Bethe, H. A., \& Brown, G. E. 1998, ApJ, 506, 780

Bethe, H. A., \& Brown, G. E. 1999, ApJ, accepted

Brown, G. E. 1995, ApJ, 440, 270

Brown, G. E. 1997, Phys. Bl., 53, 671

Brown, G. E., \& Bethe, H. A. 1994, ApJ, 423, 659

Brown, G. E., Lee, H. K., \& Wijers, R. A. M. J. 1999, in preparation

Brown, G. E., Weingartner, J. C., \& Wijers, R. A. M. J. 1996, ApJ, 463, 297

Callanan, P.J., et al. 1996, ApJ, 461, 351

Cappellaro, E., et al. 1997, A\&A, 322, 431

Chen, W., Shrader, C. R., \& Livio, M. 1997, ApJ, 491, 312

Chevalier, R.A. 1993, ApJ, 411, L33

Chevalier, R.A. 1996, ApJ, 459, 322 
Cordes, J.M., \& Chernoff, D.F. 1998, ApJ, 505, 315

de Kool, M., van den Heuvel, E.P.J., \& Pylyser, E. 1987, A\&A, 183, 47

Ergma, E., \& van den Heuvel, E.P.J. 1998, A\&A, 331, L29

Haswell, C.A., Robinson, E.L., Horne, K., Stiening, R.F., \& Abbott, T.M.C. 1993, ApJ, 411,802

Heap, S.R., \& Corcoran, M.F. 1992, ApJ, 387, 340

Kaper, L., Lamers, H.J.G.L.M., van den Heuvel, E.P.J., \& Zuiderwijk, E.J. 1995, A\&A, 300,446

Khaliullin, K.F., Khaliullina, A.I., \& Cherepashchuk, A.M. 1984, Sov. Astron. Lett., 10, 250

Langer, N. 1989, A\&A, 220, 135

MacFadyen, A., \& Woosley, S. E. 1999, ApJ, accepted

Marchenko, S.V., Moffat, A.F.J., \& Koenigsberger, G. 1994, ApJ, 422, 810

Mineshige, S., Nomoto, K., \& Shigeyama, T. 1993, A\&A, 267, 95

Mineshige, S., Nomura, H., Hirose, M., Nomoto, K., \& Suzuki, T. 1997, ApJ, 489, 227

Moffat, A.F.J., \& Robert, C. 1994, ApJ, 421, 310

Orosz, J. A., et al. 1998, ApJ, 499, 375

Portegies Zwart, S. F., Verbunt, F., \& Ergma, E. 1997, A\&A, 321, 207

Rubin, B.C., et al. 1996, ApJ, 459, 259

Ruffert, M. 1994, ApJ, 427, 351

Ruffert, M., \& Arnett, D. 1994, A\&AS, 106, 505

St.-Louis, N., Moffat, A.F.J., Lapointe, L., Efimov, Y.S., Shakhovskoy, N.M., Fox, G.K., \& Piirola, V. 1993, ApJ, 410, 342

Schaller, G., Schaerer, D., Meynet, G., \& Maeder, A. 1992, A\&AS, 96, 269

Timmes, F. X., Woosley, S. E., \& Weaver, T. A. 1996, ApJ, 457, 834

van den Heuvel, E. P. J. 1995, J. Astrophys. Ast., 16, 255 
van den Heuvel, E. P. J., \& Habets, G.M.H.J. 1984, Nature, 309, 598

Verbunt, F., \& van den Heuvel, E. P. J. 1995, in X-ray Binaries, Cambridge Univ. Press, Eds. W. H. G. Lewin, J. van Paradijs \& E. P. J. van den Heuvel, p. 457

Verbunt, F., \& Zwaan, C. 1981, A\&A 100, L7

Weaver, T. A., \& Woosley, S. E. 1993, Phys. Rept., 227, 65

Weaver, T. A., Zimmerman, G. B., \& Woosley, S. E. 1978, ApJ, 225, 1021

Wellstein, S., \& Langer, N. 1999, to be published

Wijers, R.A.M.J. 1996, Evolutionary Processes in Binary Stars, 327-344, Kluwer Acad. Publ., Eds R.A.M.T. Wijers et al.

Woosley, S. E. 1993, ApJ, 405, 273

Woosley, S. E., Langer, N., \& Weaver, T. A. 1995, ApJ, 448, 315

Woosley, S. E., \& Weaver, T. A. 1995, ApJS, 101, 181 
Table 1: Parameters of suspected black hole binaries with measured mass functions (Wijers 1996, Chen et al. 1997, Bailyn et al. 1998, Orosz et al. 1998, Bailyn, private communication). $\mathrm{N}$ means nova, XN means X-ray nova. Numbers in parenthesis indicate errors in the last digits.

\begin{tabular}{|c|c|c|c|c|c|c|}
\hline X-ray & othe & $\begin{array}{l}\text { compan. } \\
\text { type }\end{array}$ & $\begin{array}{l}P_{\text {orb }} \\
\text { (d) }\end{array}$ & $\begin{array}{l}f\left(M_{X}\right) \\
\left(\mathrm{M}_{\odot}\right)\end{array}$ & $\begin{array}{c}M_{o p t} \\
\left(\mathrm{M}_{\odot}\right)\end{array}$ & $(l, b)$ \\
\hline names & name(s) & $\begin{array}{c}\mathrm{q} \\
\left(M_{o p t} / M_{X}\right)\end{array}$ & $\begin{array}{c}K_{\text {opt }} \\
\left(\mathrm{km} \mathrm{s}^{-1}\right)\end{array}$ & $\begin{array}{c}\mathrm{i} \\
\text { (degree) }\end{array}$ & $\begin{array}{c}M_{X} \\
\left(\mathrm{M}_{\odot}\right)\end{array}$ & $\begin{array}{c}d \\
(\mathrm{kpc})\end{array}$ \\
\hline $\begin{array}{l}\text { Cyg X-1 } \\
1956+350\end{array}$ & $\begin{array}{l}\text { V1357 Cyg } \\
\text { HDE } 226868\end{array}$ & O9.7Iab & $\begin{array}{c}5.5996 \\
74.7(10)\end{array}$ & $0.25(1)$ & $\begin{array}{l}33(9) \\
16(5)\end{array}$ & $\begin{array}{c}(73.1,+3.1) \\
2.5\end{array}$ \\
\hline $\begin{array}{l}\text { LMC X-3 } \\
0538-641\end{array}$ & & B3Ve & $\begin{array}{c}1.70 \\
235(11)\end{array}$ & $2.3(3)$ & $5.6-7.8$ & $\begin{array}{c}(273.6,-32.1) \\
55\end{array}$ \\
\hline $\begin{array}{l}\text { LMC X-1 } \\
0540-697\end{array}$ & & O7-9III & $\begin{array}{c}4.22 \\
68(8) \\
\end{array}$ & $0.14(5)$ & & $\begin{array}{c}(280.2,-31.5) \\
55 \\
\end{array}$ \\
\hline$\overline{\mathrm{XN}}$ Mon 75 & 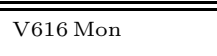 & K4 V & 0.3230 & $2.83-2.99$ & $0.53-1.22$ & $\overline{(210.0,-6.5)}$ \\
\hline A $0620-003$ & N Mon 1917 & $0.057-0.077$ & $443(4)$ & $37-44^{(\star)}$ & $9.4-15.9$ & $0.66-1.45$ \\
\hline $\begin{array}{l}\text { XN Oph } 77 \\
\text { H } 1705-250\end{array}$ & V2107 Oph & K3 V & $\begin{array}{c}0.5213 \\
420(30)\end{array}$ & $\begin{array}{c}4.44-4.86 \\
60-80\end{array}$ & $\begin{array}{l}0.3-0.6 \\
5.2-8.6\end{array}$ & $\begin{array}{c}(358.6,+9.1) \\
5.5:\end{array}$ \\
\hline $\begin{array}{l}\text { XN Vul } 88 \\
\text { GS } 2000+251\end{array}$ & QZ Vul & $\begin{array}{c}\mathrm{K} 5 \mathrm{~V} \\
0.030-0.054\end{array}$ & $\begin{array}{c}0.3441 \\
520(16)\end{array}$ & $\begin{array}{c}4.89-5.13 \\
43-74\end{array}$ & $\begin{array}{c}0.17-0.97 \\
5.8-18.0\end{array}$ & $\begin{array}{c}(63.4,-3.1) \\
2\end{array}$ \\
\hline $\begin{array}{l}\text { XN Cyg } 89 \\
\text { GS } 2023+338\end{array}$ & $\begin{array}{l}\text { V404 Cyg } \\
\text { N Cyg } 1938,1959\end{array}$ & $\begin{array}{c}\text { K0 IV } \\
0.055-0.065\end{array}$ & $\begin{array}{c}6.4714 \\
208.5(7)\end{array}$ & $\begin{array}{c}6.02-6.12 \\
52-60\end{array}$ & $\begin{array}{l}0.57-0.92 \\
10.3-14.2\end{array}$ & $\begin{array}{l}(73.2,-2.2) \\
2.2-3.7\end{array}$ \\
\hline $\begin{array}{l}\text { XN Mus 91 } \\
\text { GS } 1124-683\end{array}$ & & $\begin{array}{c}\text { K5 V } \\
0.09-0.17\end{array}$ & $\begin{array}{l}0.4326 \\
406(7)\end{array}$ & $\begin{array}{c}2.86-3.16 \\
54-65\end{array}$ & $\begin{array}{c}0.41-1.4 \\
4.6-8.2\end{array}$ & $\begin{array}{c}(295.0,-6.1) \\
3.0\end{array}$ \\
\hline $\begin{array}{l}\text { XN Per } 92 \\
\text { GRO J0422+32 }\end{array}$ & & $\begin{array}{c}\text { M0 V } \\
0.029-0.069\end{array}$ & $\begin{array}{l}0.2127(7) \\
380.6(65)\end{array}$ & $\begin{array}{c}1.15-1.27 \\
28-45\end{array}$ & $\begin{array}{c}0.10-0.97 \\
3.4-14.0\end{array}$ & $(197.3,-11.9)$ \\
\hline $\begin{array}{l}\overline{\text { XN Sco } 94} \\
\text { GRO J1655-40 }\end{array}$ & & $\begin{array}{c}\text { F5-G2 } \\
0.33-0.37\end{array}$ & $\begin{array}{c}2.6127(8) \\
227(2)\end{array}$ & $\begin{array}{c}2.64-2.82 \\
67-71\end{array}$ & $\begin{array}{l}1.8-2.5 \\
5.5-6.8\end{array}$ & $\begin{array}{c}(345.0,+2.2) \\
3.2\end{array}$ \\
\hline $\begin{array}{l}\overline{\mathrm{XN}} \\
4 \mathrm{U} 1543-47\end{array}$ & MX 1543-475 & $\mathrm{A} 2 \mathrm{~V}$ & $\begin{array}{c}1.123(8) \\
124(4)\end{array}$ & $\begin{array}{c}0.20-0.24 \\
20-40\end{array}$ & $\begin{array}{l}1.3-2.6 \\
2.0-9.7\end{array}$ & $\begin{array}{c}(330.9,+5.4) \\
9.1(11)\end{array}$ \\
\hline
\end{tabular}

( ) A much higher inclination for A0620 has been claimed by Haswell et al. (1993) of up to $\mathrm{i}=70$. In this case, the lower limits on the component masses would be $M_{X}>3.8$ and $M_{\text {opt }}>0.22$. 


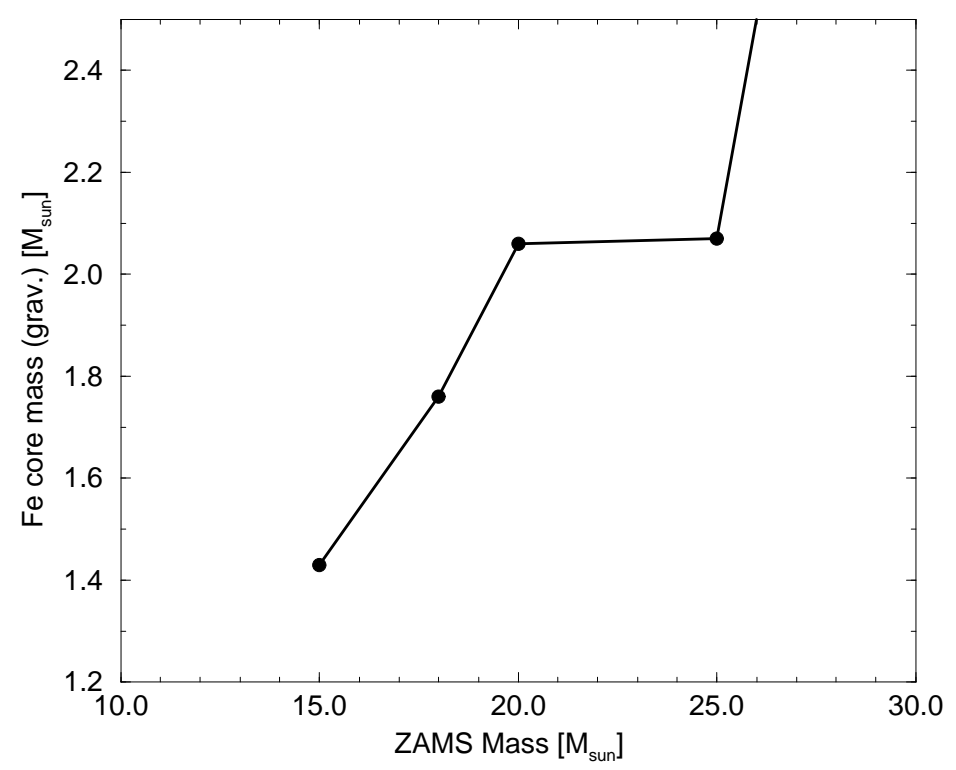

Fig. 1.- Comparison of the compact core (Remnant) masses resulting from the evolution of single stars (filled symbols), case of solar metallicity of Woosley \& Weaver (1995). 